journal club

\title{
Gibt es einen Zusammenhang?
}

Fragestellung: Ziel dieser Arbeit war der Nachweis, dass die Analyse von Biomarkern wie Alpha-Synuclein oder/und Amyloid mit der Progression der Parkinson-Erkrankung korreliert.

Hintergrund: Zu den „unmet needs“ gehört die Etablierung von Biomarkern für Parkinson. Ein Biomarker sollte erlauben, ohne teure invasive Techniken die Diagnose eines idiopathischen Parkinson-Syndroms zu stellen, das idiopathische Parkinson-Syndrom von atypischen und symptomatischen Parkinson-Syndromen zu differenzieren und/oder zusätzlich über die Progression der Parkinson-Erkrankung Auskunft zu geben.

Patienten und Methodik: Ein südschwedisches Konsortium sammelte über zwei Jahre Liquores und analysierte bei 42 Parkinson- und 69 Kontrollpatienten Alpha-Synuclein, Beta-Amyloid 1-42, Tau, phosphoryliertes Tau und Neurofilament light und korrelierte diese Daten mit dem klinischen Bild, insbesondere Alter, Geschlecht, Krankheitsdauer und Levodopa-Äquivalenzdosis. Die Kontrollpatienten waren mit durchschnittlich 74 Jahren signifikant älter als die Parkinson-Patienten mit 67,5 Jahren. Die Patienten durften keine Demenz aufweisen. Die Levodopa-Äquivalentdosis betrug im Schnitt 647,5 mg. Liquor wurde mittels Lumbalpunktion gewonnen.

Ergebnisse: Parkinson-Patienten wiesen signifikant niedrigere Alpha-Synucleinspiegel auf, auch das Tau und das phosphorylierte Tau waren im Vergleich zu Kontrollpatienten deutlich reduziert. Keiner der Biomarker in der Kontrollgruppe korrelierte mit dem Geschlecht. Höheres Alter in der Kontrollgruppe war mit höherem Neurofilament light verknüpft. Keiner der Biomarker korrelierte signifikant mit dem Ausbildungsstand der Patienten. Patienten mit längerer Krankheitsdauer und höherer Levodopa-Äquivalentdosis zeigten höhere Liquorspiegel

Hall S, Surova Y, Öhrfelt A et al. CSF biomarkers and clinical progression of Parkinson disease. Neurology 2015; 84: $1-7$ für $\mathrm{A} \beta 42$, wohingegen keine signifikanten Korrelationen zwischen Krankheitsdauer und Levodopa-Äquivalent- dosis, Tau, phosphoryliertem Tau, Neurofilament light oder Alpha-Synuclein bestanden. Liquor-Alpha-Synuclein korrelierte bei den Patienten mit Tau, phosphoryliertem Tau und Neurofilament light. Bei niedrigem A $\beta 42$ im Liquor (< $550 \mathrm{ng} / \mathrm{l})$ bei Studienbeginn verschlechterte sich die Kognition stärker als bei normalem A $\beta 42$. Auch höhere Ausgangsspiegel im Liquor für Alpha-Synuclein korrelierten mit einer stärkeren kognitiven Verschlechterung im 2-Jahres-Beobachtungszeitraum. Dagegen fanden sich keine Hinweise auf eine prognostische Aussagekraft der Ausgangsspiegel von Tau, phosphoryliertem Tau oder Neurofilament light bezüglich der kognitiven Entwicklung der Parkinson-Patienten.

Bezüglich der Korrelation der Liquormarker mit der motorischen Ausprägung der Parkinson-Erkrankung war ein hoher Ausgangsspiegel von Alpha-Synuclein im Liquor prädiktiv für eine Verschlechterung der motorischen Symptome im Beobachtungszeitraum von zwei Jahren. Auch erhöhte Spiegel von phosphoryliertem Tau im Liquor waren mit einer stärkeren Verschlechterung der motorischen Symptome assoziiert. Erhöhtes phosphoryliertes Tau korrelierte mit einem höheren Hoehn und Yahr Stadium.

Schlussfolgerungen: Hohe Ausgangswerte von Alpha-Synuclein korrelierten mit einer Verschlechterung der Kognition und motorischen Symptomatik im 2-Jahres-Beobachtungszeitraum. Auch die erhöhten phosphorylierten Tauspiegel zeigten eine gute Korrelation mit einer Verschlechterung der motorischen Symptomatik und Verschlechterung der kognitiven Leistungsfähigkeit. Ähnlich wie bei Alzheimer-Patienten war auch bei diesen Patienten ein niedriges Amyloid $\beta 42$ mit schlechterer kognitiver Leistungsfähigkeit verknüpft. Ähnlich wie in anderen Studien gezeigt, konnte diese Studie nachweisen, dass Alpha-Synuclein bei Parkinson-Patienten im Vergleich zu altersentsprechenden Kontrollpersonen reduziert ist. Dagegen konnten die Autoren zeigen, dass erhöhte Alpha-Synucleinspiegel im Liquor eine schnellere kognitive Schädigung und Störung der Motorik vorhersagen. Auch eine Verschlechterung der Motorik war mit höheren Spiegeln von phosphoryliertem Tau im Liquor verknüpft.

\section{- Kommentar von Heinz Reichmann, Dresden}

\section{Diese Ergebnisse haben (noch) keine klinischen Konsequenzen}

Diese Studie scheint nahezulegen, dass für den einzelnen $\mathrm{Pa}$ tienten gewisse Vorhersagen über die Progression der Parkinson-Erkrankung durch die Analyse von Alpha-Synuclein, phosphoryliertem Tau und $A \beta 42$ im Liquor vorgenommen werden können. Leider wird dies bis auf weiteres keine klinische Konsequenz beinhalten. Einschränkend muss auch erwähnt werden, dass die vorliegende Studie an relativ wenigen $(n=42)$ Parkinson-Patienten vorgenommen wurde und die gewonnenen Ergebnisse somit mit einem gewissen Vorbehalt betrachtet werden müssen.

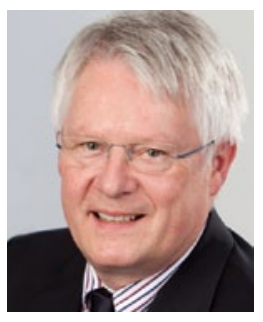

Prof. Dr. med. Heinz Reichmann, Dresden

Direktor der Klinik und Poliklinik für Neurologie, Klinikum Carl Gustav Carus der TU Dresden, Dekan der Medizinischen Fakultät. E-Mail: heinz.reichmann@ uniklinikum-dresden.de 